\title{
Review and Prospect of Research on Language Teaching Evaluation Theory in China
}

\author{
Yufang Rao \\ Wuhan University of Technology \\ Wuhan, China 430070
}

\begin{abstract}
This paper first gives a brief overview of the definition to "evaluation" of language teaching based on the analysis of basic concepts, and then summarizes the theoretical content and theoretical system of language evaluation. By reviewing the main research features and existing practical problems of the current domestic language teaching evaluation system in China, the author puts forward a multi-type and multi-level comparative analysis through linguistic libraries, which will help to improve the scientificity of evaluation and can combine the research methods of corpus with translation studies. The review and prospect of the research on the theory of domestic language teaching evaluation is helpful to give full play to the guiding and supporting role of theories in practice.
\end{abstract}

Keywords-language teaching evaluation theory; review and prospect; comparative analysis

\section{INTRODUCTION}

Based on the characteristic analysis from the perspective of human language, it can be found that description and evaluation is the basic element. The description mainly refers to that human beings originally express the conceptual cognition and depiction of the world through simple symbols; judgment mainly refers to the subjective value judgment of a person as an individual on the basis of different traits. Evaluation mainly refers to the language-based and textbased understanding of core meanings. Evaluation is indispensable in the exchange and communication among individuals. It is the main factor for judging and understanding the social significance and value of interpersonal relationships, and it is also an important part of research. The main purpose of proposing language evaluation theory is to establish and develop the language value judgment system. Beginning with analysis of the basic concept, the author briefly introduced the definition of language teaching "evaluation" before summarizing the theoretical content and system of language evaluation. By reviewing the main research features and existing practical problems of the current domestic language teaching evaluation system (hereinafter referred to as evaluation system) in China, the author proposes a multi-type and multi-level comparative analysis through linguistic libraries.

\section{BRIEF INTRODUCTION OF EVALUATION AND EVALUATION THEORY}

The understanding and definitions about "evaluation" are widely divergent and opinions in the academic circles are quite different. Li Xinyan and Liao Xu (2015) argued that the process of evaluation is the process of individual comparing and judging subjective affairs. The final evaluation result is directly related to the world view and values of the evaluating subject. It can be the evaluation following convention or free opinions against the ordinary. The way of evaluation is a common way that the participating speaker engages himself in conversations and communication situations, and then the speaker expresses his own opinions on issues and attitude. Thus, the individual's speech performance and choices are largely influenced by the ideological concepts and the social language information environment. According to the viewpoint of Peng Huilan (2015), evaluation expresses ideas of something and corresponding attitude in the form of language while constructing the language appearance of a personal with a fixed speech attitude; it is the attitudinal meaning generated in the process of interpersonal mediation and role adjustment Thus, it can be seen that, in the process of an information transmitter at the level of evaluation (including the subject of speech, the subject of writing, etc.) passing individual judgments on information recipients (including material objects and events), he/she will automatically look for group with consensus to form an alliance and meanwhile take the initiative to distance himself/herself from those with different opinions. Liu Shijun (2016) summarized and evaluated the essential attributes and the essential characteristics of things. He held that evaluation belongs to a comprehensive and complex process of cognitive comprehension, and it is also an organic whole that integrates the evaluation of the subject and the evaluation criteria. Evaluation is a dynamically changing process. Both the evaluation subject and evaluation object gradually change under the influence of subjective changing factors and may eventually be reconciled. Therefore, the study of evaluation needs to be based on the individual variables of the evaluation factors, and at the same time, the recognition of the unity of the various elements in the evaluation is also the basis for evaluation acts. The nature and attributes of evaluation also show that the behavior of evaluation is a process with strong subjective color, emotional color and 
subjective differences. To comprehensively understand the evaluation acts cannot be separated from the deep understanding of written statements and paragraph, and the deep digging of evaluation resources in existing information can be of great significance to the construction of ideology. The application of evaluation criteria in the evaluation theory can achieve the study of evaluation under different cultural backgrounds. The evaluation theory gradually obtains new meanings in the process of interpersonal meaning study and interpersonal relationship development. At the same time, it also provides a comprehensive viewing angle for the reenrichment and foundation lying of interpersonal meaning model. Interpersonal meaning comprehensively and comprehensively covers the concepts of evaluation and negotiation participation, and it is a new form expressing the attitude and emotion value of subjects in discourses. This theory first appeared and was used in the special language research project of Ableson and Abner which was conducted between 1990 and 1992. The evaluation theory is important linguistic resource. The discussion focuses on the natural negotiation and communication between the author, discourse and speaker. It is an ideological communication process in which subjects interact with each other. Ableson once stated that evaluation resources are the orientation of attitude elaboration based on semantic division, which requires speakers and narrators to express their views and attitudes directly. Precisely because of the differences between individuals' values and beliefs, there are great differences in the value judgments. Different reading individuals may interpret the same text message differently, because individuals generally forms subjective value judgments based on their own understanding. The research on evaluation theory showed that the evaluation ability and habits of an individual are the important manifestation of the individual's understanding of language and semantic meaning as well as the individual's expression potential. The evaluation manner is a common way that the participating speaker engages himself in conversations and communication situations.

\section{REVIEW OF RESEARCH ON LANGUAGE TEACHING EVALUATION THEORY IN CHINA}

Weier (2015) argued that some contents in theories of Systematic Linguistics show that the basis and soul elements of interpersonal meaning lie in tone. However, Wei Ting (2016) pointed out that, in the context of comparative analysis, it is possible to understand the inherent meaning of language evaluation, while the linguistic impact on interpersonal status cannot be ignored. The theory associated with the system of evaluation meaning is mainly the theoretical system initiated by James in the late 1980s. Since its establishment, the system has aroused widespread concern and heated discussion in the academic field of language. At present, it has gradually developed into a popular topic of the system function in linguistic research. At present, the research conducted by scholars in China on this theory can be roughly divided into two kinds: theoretical research and applied research Theoretical research mainly focuses on the evaluation of theoretical functions and their practical value and significance (Wang Huazhen, 2015; Guo
Haoran, 2015; Ma Linwei, 2016, etc.), including research on the construction value of the theory (Yang Xinzhang, 2014; Zhang Delin, 2015; Liu Shizhu, 2015). The study of application value can be divided into the academic discourse research and analysis of theoretical contents, including academic short articles, novels, editorials, speeches, interviews, etc. Nowadays, most of the scholars in China have realized the inherent limitations of application of the evaluation theory. Therefore, they have established the research viewpoints on evaluation methods and actively advocated sub-standard research and discussion of subconditions. At present, most of evaluation theories in China focus on the study of the mentality and ideology of the individuals who use the language, and there are many questions about the sentence level, the article theme and the layout of the articles. In the process of recognizing the meaning of evaluation, most scholars adopt scattered and fragmented evaluation methods in an obviously random manner, and thus it is difficult to achieve a comprehensive evaluation of the system. In the process of recognizing the evaluative meaning, attention should not only be given to the evaluation of vocabulary and ideas but should also to the symbiosis of the language system and language. Yang Xinzhang (2016) stated that the evaluation methods and means used in discourse, to some extent, can directly reflect the author's own concepts and whether the author's attitude towards his evaluation and judgment is firm or not, and meanwhile, they are also the index to assess the intensity. He also proposed that this factor can provide support to explore and study the form of ideological consciousness of the discourse. Li Zhanxin (2015) argued that, in a discourse, evaluation elements of the transitivity system can be refined from the structural features of both lengthy narrative and short paragraph, but the current evaluation system theory has not covered this part directly yet. Liu Shijun (2015) suggested that the research on evaluation theory should integrate philosophy with linguistics, so as to study the evaluation theory thoroughly from multiple perspectives. The study of evaluation theory from the perspective of philosophy should not be a single emphasis on the nature, characteristics or criteria of linguistics, because the understanding of evaluation concept and basic characteristics of language is of great importance to understand relevant concepts. Ma Lingwei (2016) pointed out that, in the crossdomain and interdisciplinary evaluation of cultural knowledge, functional grammar and additional modal verbs usually require taking into account in the analysis of discourse. However, for the moment, there is a problem of insufficient precision in grammatical analysis and evaluation, which is also an important reason limiting the scope of evaluation. The evaluation theory is the modal expressions which are not limited to the grammatical functions. They can not only achieve the inter-connection between subjects, but can also enhance the extensiveness and systematization, and ultimately maximizes the interpersonal meaning and function. Evaluation theory partly use knowledge of the socio-semiotic theory for reference, and meanwhile, this idea also further highlights the overall needs and distinctive features of social semiotics through judgments and appreciation. The main 
evaluation resources studied and analyzed in the evaluation theory achieve overall connection by the way of rhythm.

\section{Prospect OF RESEARCH ON LANGUAGE TEACHING EVALUATION THEORY IN CHINA}

Pang Weixian (2015) pointed out that expansion of the evaluation theory in interpersonal meaning mainly relies on the systematic functional theory which helps to fully interpret the morphological meaning of evaluation theory in interpersonal meaning. Research on the evaluation theory will receive more and more attention and priorities in the future, and thereby the space for its development will further expand. The deepening of the research in the field of evaluation theory and the expansion of its application scope drives the innovation of comprehensive contents and reshaping of theoretical framework in many fields such as translation and discourse analysis. It is expected that, the evaluation theory of language teaching will further go deep into the teaching of foreign language and other subjects in the future, and the prospect of theoretical application will also be brighter.

Aiming at the current limitations of the overall research on linguistic evaluation theory in China, it is needed to promote theoretical application and research through innovative combination of multiple research methods. The corpus method can be used to combine the evaluation system with contrast, translation and analysis, so as to effectively expand the scope of the subject research associated with the evaluation system.

The corpus-based evaluation theory will integrate with comparative analysis in the future. Different types of corpora should be used to realize multi-angle and multi-level analysis, and then different evaluation methods such as grammar, vocabulary and discourse can be combined to make the distribution and frequency of application between different carriers become more scientific. In the process of oral communication and using written language, it is necessary to conclude and summarize representative laws, so as to strengthen the distribution regularity of evaluation means in different styles of text. At the same time, differentiated evaluation methods and means should be applied on the same work produced by authors of different genders and ages to understand similarities and differences.

In the future, there will be a closer relationship among evaluation, evaluation subject and evaluation criteria. The evaluation criteria are influenced by many factors in the process of formulation, and therefore, it is necessary to fully recognize the evaluation differences caused by the differences in roles, perspectives and individual ideologies. Therefore, the writer and the translator need to adopt scientific evaluation methods to improve the systematic and comprehensive evaluation. The future evaluation theory will integrate with functional linguistics, corpus, translation and comparative linguistics to build a systematic evaluation system. This not only has profound meaning for deepening and expanding linguistic evaluation theory, but also provides guidance for Chinese evaluation. In the future, the evaluation theory will be widely used in many fields such as language teaching, translation, speaking and language acquisition. To the future application of the evaluation system, as mentioned above, the study of evaluation needs to be based on the individual variables of the evaluation factors while recognizing that the unity of various elements in evaluation are also the basis for evaluation of behavior. It can be seen from the nature and attributes of evaluation that the behavior of evaluation is a process with strong subjective color, emotional color and subjective differences. The deep understanding of written statements and paragraphs is essential to comprehensively understand the evaluation acts, and the deep digging of evaluation resources in existing information can be of great significance to the construction of ideology.

\section{CONCLUSION}

To sum up, this paper first studied the system of the evaluation theory and then reviewed the current state and existing problems in the research on the language teaching evaluation theory based on a clear concept. At last, the author forecasted the future development trend of the evaluation theory. The author also proposed that corpusbased evaluation theory would integrate with comparative analysis in the future. This research has some uniqueness in the subject innovation and belongs to the interdisciplinary research. It predicted the development direction of the evaluation theory scientifically through the adequate description and reasonable analysis. The multi-type and multi-level comparative analysis can be realized through linguistic database and this evaluation method will be more conducive to improving the scientific evaluation. At the same time, it is also possible to combine the research methods of corpus with the study of translation. The review and prospect of the research on the language teaching evaluation theory in China will be helpful to give full play to the guiding and supporting role of theories in practice.

\section{REFERENCES}

[1] Li Jiangnan, He Miao. Reference and Reflections of English Teaching Research on Current Southeast Asian Language Teaching [J]. Journal of Kunming Metallurgy College, 2016, 32 (06): 44-47.

[2] Zhao Xuemei. Feasibility of Applying Evaluation Theory into Oral English Teaching [J]. Brand, 2015, (01): 217-218.

[3] Lu Aihua. Analysis and Evaluation of English Textbook at Home and Abroad -- Suggestions on the Evaluation of English Teaching Materials [J]. Journal of The Chinese Society of Education, 2012, (S2): 232-233.

[4] Wen Xuemei, Yang Xiaojun. Review and Prospect of the Research on Domestic Language Teaching Evaluation Theory $[\mathrm{J}]$. Journal of Educational Science of Hunan Normal University, 2011,10 (06): 103106.

[5] Wu Xiulan. Review of Application of Formative Assessment in Foreign Language Teaching in Chinese Universities [J]. Foreign Language World, 2008, (03): 91-96.

[6] Kong Wen, Zhang Shoujin, Li Qinghua. Developing Linguistic Behavior Testing-A Review of Foreign Studies [J]. Journal of Modern Foreign Languages, 2007, (02): 200-208 + 220. 